\title{
Ear lobe crease: a marker of coronary artery disease?
}

\author{
Aris P. Agouridis ${ }^{1,2}$, Moses S. Elisaf ${ }^{1}$, Devaki R. Nair², Dimitri P. Mikhailidis ${ }^{3}$
}

${ }^{1}$ Department of Internal Medicine, Medical School, University of Ioannina, Ioannina, Greece

${ }^{2}$ Department of Clinical Biochemistry (Vascular Disease Prevention Clinics), Royal Free London Foundation Trust, Pond Street, London, UK

${ }^{3}$ Department of Clinical Biochemistry (Vascular Disease Prevention Clinics),

Royal Free Hospital Campus, University College London Medical School,

University College London (UCL), London, UK

Submitted: 14 November 2014

Accepted: 25 November 2014

Arch Med Sci 2015; 11, 6: 1145-1155

DOI: $10.5114 /$ aoms.2015.56340

Copyright $\odot 2015$ Termedia \& Banach

\section{Abstract}

The ear lobe crease (ELC) has been defined as a deep wrinkle that extends backwards from the tragus to the auricle. It has been proposed that ELC is a predictor of coronary artery disease (CAD). In this review, we consider the possible association between ELC and CAD. Our aim is to systematically address all the relevant evidence in this field. There are many studies that support an association between ELC and CAD. However, other studies did not find such an association. A recent meta-analysis supports the hypothesis that ELC could be a marker of CAD. However, several limitations raise doubts as to whether we should accept this link.

Key words: ear lobe crease, coronary artery disease, Frank's sign.

\section{Introduction}

Coronary artery disease (CAD) is a leading cause of death [1]. It is estimated that by $2030,40.5 \%$ of the US population will have some form of cardiovascular disease (CVD) and the real indirect medical cost relevant to CVD is expected to increase by $61 \%$ [1].

Several algorithms have been used to predict CAD or cardiovascular events without the use of invasive methods. These include the Framingham [2], PROCAM (Prospective Cardiovascular Münster) [3] and SCORE (Systematic COronary Risk Evaluation) [4] engines that predict risk in populations without CVD as well as the Diamond Forrester algorithm which predicts the probability of having significant CAD in symptomatic patients [5]. In a recent study, the Framingham risk score seems to outmatch the other three risk scores in 1,296 stable chest pain patients, who underwent cardiac computed tomographic angiography (CCTA) to assess CAD ( $\geq 1$ lesion with luminal stenosis of $\geq 50 \%$ ) [6]. However, the algorithms often overestimate the prevalence of CAD [7].

Coronary angiography and CCTA are used to diagnose or rule out CAD [8]. However, these invasive techniques have limitations since they involve exposure to radiation and the administration of contrast agents [9, 10]. Contrast-induced nephropathy is associated with prolonged hospitalization as well as increased cardiovascular morbidity, renal morbidity and all-cause mortality [10].
Corresponding aurthor: Dimitri P. Mikhailidis MD FRCP, FRCPath

Department of Clinical

Biochemistry

(Vascular Disease

Prevention Clinics)

Royal Free Hospital Campus

University College London

Medical School

University College London

(UCL)

Pond Street

London NW3 2QG, UK

Phone: 02078302258

Fax: 02078302235

E-mail: mikhailidis@aol.com 
There is a need for additional markers which might identify individuals at high risk of CAD. The ear lobe crease (ELC), a simple clinical sign first described by Frank in 1973 [11], could be a potential predictor of CAD. Since then, numerous studies have evaluated the potential link between ELC and risk of CAD [12-15].

In this review, we describe the possible association between ELC and CAD. Our aim is to systematically address all the relevant evidence in this field.

\section{Search criteria}

We searched PubMed up to $1^{\text {st }}$ of November 2014 using combinations of the following keywords: earlobe crease, ear lobe crease, ear-lobe crease, ear crease, ear creases, coronary artery disease and Frank's sign. Randomized controlled trials, original papers, review articles and case reports are included in the present review. References of these articles were scrutinised for relevant articles.

\section{Pathogenesis}

The ELC is a deep wrinkle, either unilaterally or bilaterally, that extends approximately $45^{\circ}$ backwards from the tragus to the auricle. Several mechanisms have been proposed to link ELC with CAD.

It seems that the prevalence of ELC increases with advancing age [16-20]; the same is true for CAD [21, 22]. The ELC is absent at birth and usually appears later in life $[23,24]$. Recently, a study involving Japanese male patients with an ELC and metabolic syndrome (a risk factor of CAD) demonstrated the presence of shortened telomeres (extreme ends of chromosomal DNA; the shortening of telomeres implies ageing) in peripheral white blood cells $[25,26]$.

In a case report, Sapira suggested a link between macrophage activity (which is involved in atherosclerosis), aging and maintaining ear lobe collagen [27].

The ELC and CAD are apparently related to the loss of elastin and elastic fibre [28]. In a subgroup analysis of 421 patients with myocardial infarction (MI) and 421 controls, biopsies of the ear lobes $(n=12)$ revealed tears of the elastic fibres in all subjects with ELC, but not in the 2 subjects with neither MI nor ELC [28].

The ELC may be a consequence of the sleeping position and in its early stages may 'resolve' during the day [23, 27, 29].

The link between ELC and CAD across different ethnic populations supports the possibility that there is a common genetic factor involved $[19,30$, 31]. However, we need to consider that this link may be less evident in some ethnicities.

\section{Epidemiology}

\section{Studies showing a positive association between ELC and CAD}

The possible association between ELC and CAD was first described by Frank in 1973, when he observed that 19 out of 20 patients with an ELC had at least one of the known risk factors for CAD [11]. Small studies with $<10$ subjects suggested an association between ELC and CAD [32-38].

The first controlled study that examined the relationship between ELC and CAD was carried out in a USA coronary-care unit [39]. Lichstein et al. found that $47 \%$ of 531 patients from New York who had acute MI had ELC (unilateral or bilateral), which was significantly greater than the $30 \%$ observed among 305 age-matched control subjects with no clinical evidence of CAD $(p<0.001)$ [39].

Others (publishing in the form of letters to editors) have also supported the possible association between ELC and CAD [40, 41]. Christiansen et al. examined the prevalence of diagonal ELC among 523 Danes (320 men) admitted to a medical-surgical unit [40]. The prevalence of ELC in patients with an acute $\mathrm{Ml}$ aged from 50 to 59 (46.8\%) was significantly greater than in an age-matched control group (31.6\%) [40]. Similar results were obtained in a group aged 60 to 69 years but not in those aged $70-79$ years and $>80$ years. The investigators concluded that a significant correlation was found between ELC and both CAD $(p<0.02)$ and increasing age of the patients $(p<0.0001)$. Of note, a stronger correlation was found between CAD and ELC than between CAD and other risk factors such as hypertension, smoking and diabetes [40].

In an autopsy study, Lichtstein et al. found a positive correlation between diagonal ELC and severe CAD. Subjects with bilateral ELC had more severe CAD than those without ELC $(p<0.01)$ and with unilateral ELC [41].

In 1978, Kaukola reported that $69 \%$ of the men and $69 \%$ of the women among 219 patients from Finland (165 men, age range: 32-65 years) who sustained an MI had a diagonal ELC. The prevalence of ELC was significantly greater when compared with the prevalence of $24 \%$ in a group of 290 healthy, similarly aged subjects (236 men) [42].

In another Finnish study by Kaukola et al. [30], from 286 patients (age range: 26-66) who underwent coronary angiography because of typical symptoms or signs of CAD or atypical chest pain, 200 (174 men; mean age: 48 ) had $\geq 50 \%$ stenosis (CAD) in $\geq 1$ coronary artery and 86 individuals (35 men; mean age: 50 years) were without any significant stenosis $(\leq 50 \%)$. The prevalence of ELC in the group with CAD was $72 \%$, whereas in the group without CAD it was only $21 \%(p<0.001)$. The authors [30] concluded that the prevalence of 
diagonal ELC increases with age and severity of CAD but showed no correlation between ELC and other coronary risk factors such as high cholesterol, high triglycerides, low high-density lipoprotein cholesterol, hypertension, smoking, diabetes and obesity [30].

In 1982, the same author [43] examined the possibility that ELC is inherited. He evaluated 41 male patients (mean age: 44 years) with previous $\mathrm{Ml}$ and their 134 first-degree relatives, in order to document the prevalence of ELC [43]. The ELC was present in $59 \%$ of the patients with MI. From the 74 relatives of this group, those with CAD, approximately $50 \%$ of the parents, approximately $25 \%$ of healthy brothers and $31 \%$ of healthy sisters also had ELC. None of the children had ELC. Kaukola concluded that those results do support an association between ELC and CAD but do not indicate that ELC is an inheritable sign [43].

Sprague examined 222 patients in the USA, who were scheduled for elective surgery [17]. He found that ELC increased with age as only 3 patients out of $120<40$ years old had the sign whereas the sign was visible in 61 out of the remaining 102 patients who were older than 40 years $(p<0.0001$, by our calculation, Fisher's test). Among those 102 patients, 50 had CAD. The prevalence rate of ELC in patients with CAD was $82 \%$, and this was significantly higher than the $38.5 \%$ observed in the remaining 52 patients without CAD ( $p<0.0001$, by our calculation, Fisher's test). Sprague concluded that ELC was positively related with CAD, age and increased complications of anaesthesia [17].

Doering et al. compared 50 patients with CAD with 38 patients without CAD in the USA on the basis of age, weight, smoking history, diabetes and the presence of an ELC. All the above factors were correlated with $C A D$, but the strongest correlation was noted between CAD and ELC $(p<0.001)$ [44].

Kristensen found a positive correlation between bilateral diagonal ELC and CAD in 74 male hypertensive Danes when compared with 29 normotensive control age-matched males $(p<0.005)$ [45]. On the other hand, no genetic factor seemed to be involved in the cause and development of the ELC [45].

A very strong relationship between ELC and CAD was found in a Polish study where 72 patients out of 160 with CAD and only 23 patients out of 340 without CAD had ELC $(p<0.00001)$ [46].

In a study from Israel, Shoenfeld et al. found a higher prevalence $(77 \%)$ of ELC in 421 patients with $\mathrm{MI}$ when compared with a $40 \%$ prevalence rate of 421 controls $(p<0.05)(p<0.0001$, by our calculation, Fisher's test), regardless of age [28]. Moreover, the prevalence of ELC was even higher in $\mathrm{Ml}$ patients with diabetic retinopathy, hyper- tension, and in Ashkenazi Jews compared with non-Ashkenazi Jews [28].

Pasternac and Sami [18] assessed 340 Canadian patients who underwent coronary arteriography; $75.6 \%$ of them had CAD. The prevalence of CAD in patients with ELC was $91.1 \%$. This was significantly higher $(p<0.001)$ when compared with the $60.5 \%$ for patients without ELC. Of note, the positive predictive value of ELC in men was $95.7 \%$ while in women it was much lower (66.7\%) [18]. The authors concluded that ELC has a high predictive value for CAD, but its absence does not rule out such a disease [18].

In a large study, Elliot suggested that ELC should be regarded as a risk factor of CAD [47]. Among 1000 patients who were admitted to large urban medical centres in St. Louis, Missouri (USA), ELC was present in 275 out of 376 patients with $C A D$, whereas it was seen in only 98 out of 624 patients without CAD $(p<0.00001)$ [47]. Similar results were seen in a subgroup analysis of the above study; among 205 patients who underwent coronary angiography 121 out of 148 patients with CAD had ELC [47]. In another prospective, subgroup analysis of the same study, in 112 patients who underwent cine coronary angiography only ELC and previous MI were found to significantly correlate with CAD proved by angiography ( $p<0.00001$ and $p<0.002$, respectively) [47]. Of note, patients were considered as having CAD if coronary angiography showed $>75 \%$ stenosis of $\geq 1$ major coronary artery and if they had an acute $\mathrm{MI}$ or history of angina [47].

Similar results were obtained in 1000 Japanese patients (573 men) [48]. The ELC was present in 58 out of 237 (24.5\%) patients with CAD, a prevalence which was significantly greater than that of 35 out of 720 (4.8\%) patients without CAD $(p<0.001)$ [48]. In addition, in a subgroup analysis of the above study, in 200 patients who underwent coronary angiography ELC was present in 31 out of $119(26.1 \%)$ patients with $>50 \%$ stenosis of $\geq 1$ major coronary artery but in only 3 out of 81 (3.7\%) without CAD $(p<0.01)$ [48]. The ELC was also found to correlate significantly with male sex, age > 50 years, hypertension and smoking. However, in a multivariate analysis in the 200 patients who underwent coronary angiography, CAD and age $>50$ years were significantly related to the presence of ELC while the other risk factors appeared to be unrelated [48]. The authors concluded that, apart from the increased prevalence of ELC with advancing age, ELC is an independent predictor for CAD in Japanese adults. Of note, the prevalence of ELC in this population is very low when compared with the results of studies in Europe and America [48].

The overall incidence of bilateral diagonal ELC in 350 non-selected patients was 45\%, with 
a significant increase over the age of 50 years old $(p<0.001)$ [49]. The CAD was present in $65 \%$ of patients with ELC, a prevalence that was significantly greater than that of $23 \%$ of patients without ELC $(p<0.001)$. In addition, it was shown that diagonal ELC is significantly associated with hypertension ( $p$ $<0.01$ ), sex (higher incidence of ELC was observed in males $(66 \%)$ than in females $(34 \%),(p<0.02))$, smoking $(p<0.05)$ and hypercholesterolaemia $(p<0.05)$, but no relationship was observed with diabetes mellitus and obesity [49].

Lesbre et al. found a significant association $(p<0.001)$ between ELC and CAD (> 75\% stenosis in $\geq 1$ main coronary artery) in 172 patients [50]. Sensitivity, specificity and positive predictive value of ELC for detecting CAD were 75, 57.5 and $80.3 \%$, respectively. The authors concluded that ELC should be considered as a marker of CAD, independently of other risk factors [50].

In an autopsy study, Cumberland et al. examined the relationship between ELC and severe CAD ( $>75 \%$ stenosis of $\geq 1$ major coronary artery) in 800 patients in the USA [51]. The authors found that the presence of ELC was significantly correlated with severe CAD $(p<0.01)$ and that the absence of ELC is a predictor of the absence of severe coronary atherosclerosis [51].

Similar results were seen in 376 postmortems (206 men) carried out in the UK [52]. The risk of death from $\mathrm{MI}$ in men with ELC was 2.50 whereas in women it was 3.70. In addition, the relative risk of a male with bilateral diagonal ELC having severe CAD ( $>75 \%$ stenosis of a coronary artery) was 1.64 and for females it was 3.65. The sensitivity and specificity of bilateral diagonal ELC for detecting severe CAD were $62.1 \%$ and $65.9 \%$ for men and $69.2 \%$ and $78 \%$ for women, respectively. The authors concluded that ELC could be a useful sign of atherosclerosis [52].

In another necropsy study in the UK, Kirkham et al. found a strong association between ELC and a cardiovascular cause of death in men and women after age, height, and diabetes had been adjusted for [53].

In an Indian population, Verma et al. reported that bilateral diagonal ELC was significantly more pronounced in patients with documented CAD $(p<0.001)$ and that the prevalence of ELC increases with advancing age $[16,54]$. Of note, the combined presence of ELC and ear canal hair represented a more sensitive index of CAD [16, 54].

In 1989, in the Copenhagen City Heart Study, 14223 healthy persons were followed for 6.5 years in order to determine the relationship between diagonal ELC and the development of a first acute $\mathrm{MI}$ [55]. Of note, the right ELC was examined. After adjusting for age and sex, the authors concluded that those with ELC had a risk of acute
MI, 1.4 times greater than those without [55]. The same team 2 years later reported similar results [56]. In addition, in a recent (2014) prospective cohort study, Christoffersen et al. followed up the same population until 2011 [57]. The authors concluded that visible signs such as ELC, male pattern baldness and xanthelasmata, alone or in combination, are associated with increased risk of CAD and $\mathrm{MI}$ in the general population, independent of age and cardiovascular risk factors [57].

Miric et al. found that among 243 patients from Croatia, ELC was present in $72.7 \%$ of 143 with proven CAD and in $48 \%$ of the remaining 100 patients without CAD $(p<0.001)$ [58]. Additionally, ELC was more prevalent in patients older than 50 years than in younger patients [58].

In a Japanese autopsy study, where 100 men aged from 50 to 79 years old, who died free of vascular diseases or related conditions, were studied, Ishii et al. concluded that ELC provides a significant external marker for atherosclerosis [59].

In a prospective, cohort study which was conducted in St. Louis (USA), 108 patients were followed up for 8 to 10 years in order to ascertain whether the diagonal ELC is associated prospectively with future death or cardiac events [60]. During that time 58 deaths occurred. The prevalence of death due to acute $\mathrm{MI}$, sudden cardiac death or heart failure was greater in patients with ELC whether they had CAD $(p=0.008)$ or not $(p<0.001)$ compared with patients without ELC [60]. In addition, the prevalence of cardiac events such as cardiac death, nonfatal MI, or coronary artery bypass surgery was also higher in patients with ELC whether they had CAD $(p=0.009)$ or not $(p<0.001)$ compared with those without ELC [60]. In this study, Elliott and Karrison suggested that diagonal ELC is associated with increased all-cause and cardiac morbidity and mortality [60]. Apart from the above findings, Elliott and Powel additionally demonstrated for the first time in a living population (264 patients) a graded association between the number of creased ears (i.e. unilateral or bilateral) and future coronary risk [61].

In a Brazilian study of 1464 patients (760 men), Tranchesi et al. found that ELC was present in 220 out of 338 patients (65\%) with CAD (> 70\% stenosis of $\geq 1$ coronary artery documented by angiography), and this prevalence was significantly greater when compared with the $28 \%$ of 1086 patients without CAD ( $p<0.0001)$ [62]. In addition, the sensitivity of ELC was $65 \%$, the specificity $72 \%$, the positive predictive value $42 \%$ and the negative predictive value $87 \%$ for the diagnosis of CAD. Of note, ELC was more prevalent in the white $(41 \%)$ than in the non-white population (23\%). However, the prevalence of ELC in both populations was significantly greater in patients 
with CAD [62]. Moreover, the presence of ELC was also related to the severity of CAD. When the stenosis was present in 1 to 3 coronary arteries, the prevalence of ELC increased from $55 \%$ to $78 \%$ $(p=0.015)$ [62].

The association between ELC and CAD was found to be significant in 247 patients admitted to an acute general hospital in Dublin, Ireland [63]. The prevalence of ELC did not differ between males and females. In addition, no correlation was found between ELC and smoking, hypercholesterolaemia, hypertension, peripheral arterial disease or stroke.

Similar results were found in a non-cardiac patient population in London [64]. Motamed et al. reported a significant association between ELC and a history of CAD and that the ELC and CAD correlation was higher than that of CAD and other risk factors. However, the sensitivity, specificity and positive predictive value of the sign was $48 \%$, $88 \%$ and $16 \%$, respectively. These findings are of little clinical importance [64].

In a Croatian case-control study, Miric et al. found that diagonal ELC was present in $62.9 \%$ of 842 men ( $<60$ years old) admitted for the first non-fatal MI, and that this prevalence was greater than that of $46 \%$ of 712 age-matched controls admitted with non-cardiac diagnoses and without clinical signs of CAD [65]. The relative risk ratio $(95 \% \mathrm{Cl})$ of $\mathrm{MI}$ for men with ELC was 1.37 (1.25-1.5). This did not change when it was adjusted for age and other risk factors [65]. The authors concluded that dermatological signs such as baldness, thoracic hairiness and diagonal ELC indicate an additional risk of $\mathrm{MI}$ in men under the age of 60 years, independently of age and other established coronary risk factors [65]. One of the authors of the above study suggests that the presence of these dermatological signs (ELC, baldness, hair greying and wrinkling of the skin) in patients could be indicators of atherosclerosis [66].

Kuri et al. evaluated the usefulness of the ELC sign as a marker of CAD in 530 Japanese patients, aged $>40$ years old, who were undergoing elective surgery [67]. The authors concluded that ELC may be a useful marker for the presence of CAD in patients for whom little or no history and investigations are available [67].

In a large study in Turkey, 3722 individuals (1250 males) were evaluated for the presence of ELC in diseases such as diabetes mellitus, hypertension, MI and CAD [68]. The authors suggested that ELC could be a valuable sign in diagnosing and screening such diseases. Of note, ELC was more pronounced in males than in females [68].

In 2004, also in Turkey, Evrengül et al. examined 415 patients (306 men) for the presence of bilateral ELC. There was a greater prevalence of
ELC in 296 patients with documented CAD (> 70\% stenosis of 1 of the 3 major coronary arteries) by angiography (51.4\%) than in 119 patients (15.1\%) without CAD $(p<0.0001)$ [69]. Sensitivity of bilateral ELC was $51.3 \%$, specificity was $84.8 \%$, the positive predictive value was $89.4 \%$ and the negative predictive value was $41.2 \%$ for the diagnosis of CAD. The ELC was an independent variable for CAD. Of note, the presence of bilateral ELC was significantly associated not only with CAD but also with other coronary risk factors such as hypertension, male gender and smoking. There was no association between ELC, diabetes, lipidaemia and obesity. The authors concluded that bilateral ELC might be a useful sign in clinical practice [69].

In a Swedish autopsy study, $55 \%$ of 520 subjects (420 males, mean age 56) had diagonal ELC [70]. There was no significant difference between the presence of ELC in males (53.8\%) and females (60\%). The ELC was unilateral in $8.8 \%$ and bilateral in $91.2 \%$ of the subjects. The prevalence of ELC was $65.4 \%$ in those with CAD and $73.3 \%$ in victims of sudden cardiac death, a difference which was significant when compared with $46.1 \%$ and $57.9 \%$, respectively in the control groups [70]. The sensitivity, specificity and positive and negative predictive values for those $<40$ years old were $0.68,0.84,0.80,0.72$, respectively; for those between ages 40 and 50 years old, they were 0.68 , $0.60,0.63,0.66$, respectively; and for those over 60 years old they were $0.79,0.33,0.51,0.61$, respectively [70]. The authors concluded that ELC could be useful in screening for premature CAD in younger individuals [70]. Of note, there was a significantly greater heart weight in patients with ELC than in those without ELC $(p<0.0001)$ and in those dying from sudden cardiac death compared with deaths from other causes $(p<0.0001)$ [70].

In a Spanish study, Lamot et al. found a significant relation between the presence of a diagonal ELC and CAD and suggested that this sign could be useful in clinical practice, mainly among patients aged between 30 and 60 years [71].

In a recent study carried out in the USA, the relation between diagonal ELC and CAD was examined in 430 patients without history of CAD who underwent coronary angiography [72]. Severe CAD was documented when $>50 \%$ stenosis was found in $\geq 1$ coronary artery in the angiography. The CAD was categorized as any CAD, significant $C A D, \geq 2$ diseased vessels and $\geq 3$ diseased segments. All categories of CAD were more prevalent in the group with diagonal ELC than in the group without diagonal ELC $(p<0.001$, for all comparisons). Diagonal ELC was present in $71 \%$ of all the patients and, similarly, the prevalence of any CAD was $71 \%$ of all the patients. The prevalence of any CAD in 307 patients with diagonal ELC was 
$77 \%$. This was significantly greater when compared with that of $55 \%$ of 123 patients without diagonal ELC ( $p<0.001)$. In addition, sensitivity, specificity, positive and negative predictive values for diagonal ELC to diagnose any CAD were 78, 43, 77 and $45 \%$, respectively [72]. Of note, Shmilovich et al. were the first to report that diagonal ELC is associated with the presence, extent, and severity of CAD and that this association remained even after adjusting for age, male gender and other risk factors for CAD [72].

The same author, in a more recent study, probably in the same population as for the above study, found that diagonal ELC is a superior predictor of CAD (> 50\% stenosis of coronary arteries) than the Diamond Forester algorithm [73]. The authors suggested that, in patients with chest pain, the combination of the diagonal ELC and Diamond-Forester algorithm was a better predictor of CAD than each alone [73].

In a Chinese study of 100 patients, the prevalence of ELC in 50 patients with CAD was significantly greater than in those without $C A D$ $(p<0.05)$ [74]. The sensitivity, specificity and positive predictive value for diagonal ELC to diagnose CAD were $61 \%, 58 \%$ and $59.3 \%$, respectively [74]. The authors suggested that ELC was associated with the presence of CAD [74].

In a Chinese cohort study, among 449 consecutive Chinese patients who underwent coronary artery angiography, the prevalence of diagonal ELC was $75.2 \%$ in 250 patients with CAD, which was significantly greater than that of $46.2 \%$ of 199 patients without CAD $(p<0.001)$ [75]. Moreover, sensitivity, specificity, positive and negative predictive values for diagonal ELC to diagnose CAD in the whole population were $75.2 \%, 53.8 \%, 67.1 \%$ and $63.3 \%$, respectively. Diagonal ELC remained a positive predictor for CAD even after adjusting for age, gender and traditional risk factors, but not for hypertension, diabetes mellitus, hypercholesterolaemia and hypertriglyceridaemia. The authors concluded that there is a significant association between diagonal ELC and CAD independent of established risk factors among Chinese people [75].

\section{Studies showing no association between ELC and CAD}

Several studies have reported no significant correlation between ELC and CAD. The first report was an American study of 211 patients undergoing coronary angiography in which no significant association between ELC and CAD was found [76].

In a letter to the editor, Burton refers to a British study which found no significant difference between the presence of bilateral ELC in $52 \%$ of 48 males with $\mathrm{MI}$ and in $58 \%$ of 48 age-matched controls [77].
In 1977, among 1237 Japanese-American men, the prevalence of ELC was $30 \%$ in 71 patients with any CAD (angina, coronary insufficiency and MI) $22 \%$ in 37 patients with $\mathrm{Ml}$ and in $32 \%$ of patients without CAD. Rhoads et al. concluded that no significant relation was found between ELC and CAD in Japanese-American men [78, 79].

Similar results were obtained in 216 Native American Indians, where no correlation between ELC and CAD was found.

In a small controlled study (Northern Ireland) which included 23 patients with acute $\mathrm{MI}$ and 23 matched controls, Farrell and Gilchrist found no correlation between ELC in the occurrence of MI [80].

Jorde et al. reported lack of an association between ELC and coronary risk factors such as hypertension, diabetes mellitus, smoking and obesity in 686 Americans [81].

Moreover, no significant correlations were found between ELC and CAD in 100 adult Caucasian (Vermont, USA) patients (68 men) with the clinical diagnosis of aortic stenosis who underwent coronary angiography [82]. The authors concluded that ELC is of little clinical importance [82].

In 261 consecutive men who underwent coronary angiography, ELC was present in $67 \%$ of this population [83]. The prevalence of CAD in 175 men with ELC was $85 \%$ and in 86 men without ELC it was also $85 \%$. The authors found no correlation between ELC and CAD and concluded that their association is justifiable due to the fact that ELC and CAD both increase with age [83].

In several articles, Cheng refers to a large study from China which concluded that ELC has no predictive value for CAD in 3155 persons; ELC was attributed to age [84-86].

In a prospective Irish study, among 125 consecutive patients who underwent coronary angiography, ELC was present in 65 patients [87]. The authors found no relation between ELC and age, sex, smoking, previous $\mathrm{MI}$, hypertension, family history of heart disease, body mass index or angiographically defined CAD. In this context, the authors concluded that ELC is not a marker of CAD [87].

In an Austrian prospective clinical investigation, 233 patients with CAD were evaluated [88]. Eber and Delgado suggested that the presence and extent of diagonal ELC may not be regarded as a general prognostic factor for coronary atherosclerosis [88].

Kuon et al. assessed 670 German patients who underwent coronary angiography. The prevalence of CAD (> 70\% stenosis of coronary arteries) was 55\% in patients with ELC and $55.9 \%$ in patients without ELC. No significance was found. The authors concluded that although ELC is associated with age and overweight, it does not predict CAD [89]. 
In a subgroup analysis of the Fremantle Diabetes Study, which included 1022 diabetic patients, the prevalence of ELC was 55\% [90]. Although patients with an ELC were more likely to have CAD than those without an ELC ( $p=0.019)$, the sensitivity, specificity, positive predictive value and negative predictive value of ELC for detecting CAD were $60,48,37$ and $71 \%$, respectively. After adjusting for coronary risk factors, the authors concluded that ELC is not a predictor of CAD [90]. Similar conclusions were reached concerning diabetic retinopathy [90].

In 2009, Koracevic and Atanaskovic reported their unpublished data from a study which they had conducted 13 years before in Serbia [91]. They evaluated 78 hospitalized patients (60 men) with mean age 60.4 years. They concluded that whether or not patients had unilateral or bilateral diagonal ELC, or whether the crease was deep or superficial, it did not represent a marker of CAD [91].

\section{Ear lobe crease and other diseases}

Numerous other studies have tried to associate ELC with other diseases or coronary risk factors such as hypertension, diabetes, stroke, metabolic syndrome, peripheral arterial disease (PAD), carotid intima-media thickness (cIMT), obesity, stress and alcohol use. Park et al. found a strong correlation between ELC and haemorrhagic stroke [92]. More specifically, ELC was independently associated with subarachnoid haemorrhage and intracerebral haemorrhage [92]. In a recent case report, ELC was observed in a patient with sub-acute occipital infarction in the left posterior cerebral artery [93]. Others suggested or tried to correlate ELC with stress [94], anxiety [95], schizophrenia and bipolar disorder [96]. Several studies significantly correlated the presence of ELC with hypertension $[28,69,97,98]$, while others failed to find a positive correlation of ELC with hypercholesterolaemia [99]. Furthermore, others reported an association of ELC with metabolic syndrome [25, $100]$ or obesity $[78,79,89]$. Controversial results have been produced regarding the association of ELC with diabetic retinopathy [28, 101, 102]. Of note, a negative association was found between the presence of ELC and alcohol use in 625 white women [103]. Interestingly, in several reports the presence of ELC was significantly correlated with cIMT [104-106], whereas only one study did not reach significance [107]. Pieri et al. reported 2 histological lesions in the ear lobes of diabetic patients: perivascular cellular infiltration and thickening of adventitia reticularis in arterioles, precapillaries, capillaries and venules [108]. Several studies suggested using an arterialized ear lobe blood sample for the measurement of arterial $\mathrm{O}_{2}$ pressure $\left(\mathrm{pO}_{2}\right)$ while others indicated that arteri- alized ear lobe blood underestimates the real value [109-111]. Recent studies found a significant correlation of ELC with PAD $[112,113]$. In addition, ELC was found to have a significant correlation with brachial-ankle pulse wave velocity [114], and with the cardio-ankle vascular index [115].

\section{Comment}

It is difficult to explain why there is so much variation in the studies described above. Even 41 years after the ELC sign was first described [11] there is still uncertainty regarding its relevance.

It is important to establish whether ELC is a coronary risk factor and whether this physical sign can improve vascular risk assessment. Although the majority of studies suggested an association between ELC and CAD, there are some limitations. Age, gender, race, and ear lobe shape should not be neglected if we want to evaluate the validity of ELC as a predictor of CAD. A study by Overfield and Call [19] examined 324 healthy adult subjects (70 south western Alaskan Eskimos, 167 Navajos and 87 whites). The authors found that ELC is more frequent with age in healthy adults, creasing is related to the shape of the ear lobe, and the age of onset of creasing is influenced by race [19].

The mechanisms leading to the concurrent development of ELC and CAD remain uncertain. Ethnic differences may play an important role in the prevalence of ELC. Petrakis and Koo evaluated the prevalence of ELC in various racial groups using police arrest photographs [31]. They found an age-related increase in the frequency of ELC in White, Black and Latin-American males but no increase among the Chinese and Hawaiian-Samoans [31]. It should also be considered that ELC may only predict CAD within a limited age range [17-19]. This is because the ELC is absent at birth and rarely seen in the young, whereas it is more common in the elderly $[23,24]$.

The presence of the ELC may be influenced by the time of the day [27]. This is because the ELC may become more apparent after sleeping and distorting the ear lobe. This factor may need to be taken into account when the prevalence of ELC is assessed.

Medication administered to treat vascular risk factors (e.g. statins) can delay, arrest or even reverse CAD progression [116], even in the elderly [117]. Does such treatment (or lifestyle measures such as smoking cessation) also affect the progression/development of ELC? If so, then studies including patients on such treatment would have some limitations. This possibility has to be considered in the light of evidence that risk factors (hypertension, smoking, hypercholesterolaemia and diabetes) are associated with an increased 
prevalence of ELC, at least in some studies (as discussed above).

A large meta-analysis that investigated the possible association of ELC with CAD was recently published [118]. In this meta-analysis which included >31,100 patients, the overall sensitivity was $62 \%$ and the specificity was $67 \%$. Thus, $62 \%$ of patients with CAD would be expected to have ELC, while $67 \%$ of those without CAD would be expected not to have ELC. In addition, the risk of CAD is 3.3-fold higher in patients with ELC compared with those without ELC, a conclusion that suggests ELC as a marker for CAD [118].

We also need to assess any relationship between ELC and factors affecting arterial inflammation [119] or elastance [120]. Both these processes may be involved in the pathogenesis of ELC and vascular disease. Additional markers such as metalloproteinases [121] should also be considered in the pathogenesis of CAD and ELC. There is also a need to explore the relationship between various risk engines and the presence of ELC.

\section{Conclusions}

Large-scale clinical studies are needed to reach more robust conclusions regarding the clinical significance of ELC as a predictor of CAD. Future studies should consider the variables of age, race and ear lobe shape. In addition, these studies should also address whether there are common features involved in the pathogenesis of ELC and CAD, as little is known in this field. Moreover, prospective cohort studies should follow up the newly diagnosed patients with CAD and observe any changes in their ear lobes as the disease progresses. In this context, a grading system for ELC has been proposed [82].

After taking all the evidence into consideration, we cannot state with confidence that ELC represents a marker of CAD. However, we suggest that patients with ELC may benefit from being monitored more closely for the potential presence of CAD.

\section{Conflict of interest}

This review was written independently. No company or institution supported it financially. Some of the authors have given talks, attended conferences and participated in trials and advisory boards sponsored by various pharmaceutical companies.

The authors declare no conflict of interest.

\section{References}

1. Heidenreich PA, Trogdon JG, Khavjou OA, et al. Forecasting the future of cardiovascular disease in the United States: a policy statement from the American Heart Association. Circulation 2011; 123: 933-44.
2. D’Agostino RB Sr, Vasan RS, Pencina MJ, et al. General cardiovascular risk profile for use in primary care: the Framingham Heart Study. Circulation 2008; 117: 743-53.

3. Assmann G, Cullen P, Schulte H. Simple scoring scheme for calculating the risk of acute coronary events based on the 10-year follow-up of the prospective cardiovascular Munster (PROCAM) study. Circulation 2002; 105: 310-5.

4. Conroy RM, Pyorala K, Fitzgerald AP, et al. Estimation of ten-year risk of fatal cardiovascular disease in Europe: the SCORE project. Eur Heart J 2003; 24: 987-1003.

5. Diamond GA, Forrester JS. Analysis of probability as an aid in the clinical diagnosis of coronary-artery disease. N Engl J Med 1979; 300: 1350-8.

6. Versteylen MO, Joosen IA, Shaw LJ, et al. Comparison of Framingham, PROCAM, SCORE, and Diamond Forrester to predict coronary atherosclerosis and cardiovascular events. J Nucl Cardiol 2011; 18: 904-11.

7. Cheng VY, Berman DS, Rozanski A, et al. Performance of the traditional age, sex, and angina typicality-based approach for estimating pretest probability of angiographically significant coronary artery disease in patients undergoing coronary computed tomographic angiography: results from the multinational coronary CT angiography evaluation for clinical outcomes: an international multicenter registry (CONFIRM). Circulation 2011; 124: 2423-32, 1-8.

8. Min JK, Shaw LJ, Devereux RB, et al. Prognostic value of multidetector coronary computed tomographic angiography for prediction of all-cause mortality. J Am Coll Cardiol 2007; 50: 1161-70.

9. Mark DB, Berman DS, Budoff MJ, et al. ACCF/ACR/AHA/ NASCI/SAIP/SCAI/SCCT 2010 expert consensus document on coronary computed tomographic angiography: a report of the American College of Cardiology Foundation Task Force on Expert Consensus Documents. J Am Coll Cardiol 2010; 55: 2663-99.

10. Katsiki N, Athyros VG, Karagiannis A, et al. Contrast-induced nephropathy: an "all or none" phenomenon? Angiology 2015; 66: 508-13.

11. Frank ST. Aural sign of coronary-artery disease. N Engl J Med 1973; 289: 327-8.

12. Friedlander $\mathrm{AH}$, Scully $\mathrm{C}$. Diagonal ear lobe crease and atherosclerosis: a review of the medical literature and oral and maxillofacial implications. J Oral Maxillofac Surg 2010; 68: 3043-50.

13. Friedlander AH, Lopez-Lopez J, Velasco-Ortega E. Diagonal ear lobe crease and atherosclerosis: a review of the medical literature and dental implications. Med Oral Patol Oral Cir Bucal 2012; 17: e153-9.

14. Schilling WH, Crook MA. Cutaneous stigmata associated with insulin resistance and increased cardiovascular risk. Int J Dermatol 2014; 53: 1062-9.

15. Dwivedi S, Jhamb R. Cutaneous markers of coronary artery disease. World J Cardiol 2010; 2: 262-9.

16. Verma SK, Khamesra R, Mehta LK, et al. Ear-lobe crease and ear-canal hair as predictors of coronary artery disease in Indian population. Indian Heart J 1989; 41: 86-91.

17. Sprague DH. Diagonal ear-lobe crease as an indicator of operative risk. Anesthesiology 1976; 45: 362-4.

18. Pasternac A, Sami M. Predictive value of the ear-crease sign in coronary artery disease. Can Med Assoc J 1982; 126: 645-9.

19. Overfield T, Call EB. Earlobe type, race, and age: effects on earlobe creasing. J Am Geriatr Soc 1983; 31: 479-81.

20. Dharap AS, Sharma HS, Than M. Ear lobe crease: incidence in a healthy Malay population. Anthropol Anz 2000; 58: 309-15. 
21. Berthold HK, Gouni-Berthold I. Lipid-lowering drug therapy in elderly patients. Curr Pharm Des 2011; 17 : 877-93.

22. Athyros VG, Katsiki N, Tziomalos K, et al. Statins and cardiovascular outcomes in elderly and younger patients with coronary artery disease: a post hoc analysis of the GREACE study. Arch Med Sci 2013; 9: 418-26.

23. Williams RR. Myocardial infarction risk, earlobe crease, and sleep apnoea syndrome. Lancet 1989; 2: 676-7.

24. Merlob P, Amir J, Reisner SH. Diagonal earlobe crease in newborn infants and in children. Clin Pediatr (Phila) 1981; 20: 739-40.

25. Higuchi Y, Maeda T, Guan JZ, et al. Diagonal earlobe crease are associated with shorter telomere in male Japanese patients with metabolic syndrome. Circ J 2009; 73: 274-9.

26. Tzanetakou IP, Katsilambros NL, Benetos A, et al. "Is obesity linked to aging?": adipose tissue and the role of telomeres. Ageing Res Rev 2012; 11: 220-9.

27. Sapira JD. Earlobe creases and macrophage receptors. South Med J 1991; 84: 537-8.

28. Shoenfeld Y, Mor R, Weinberger A, et al. Diagonal ear lobe crease and coronary risk factors. J Am Geriatr Soc 1980; 28: 184-7.

29. Brumfield DT. Diagonal earlobe creases. West J Med 1984; 140: 793.

30. Kaukola S, Manninen V, Valle M, et al. Ear-lobe crease and coronary atherosclerosis. Lancet 1979; 2: 1377.

31. Petrakis NL, Koo L. Earlobe crease. Lancet 1980; 1: 376.

32. Frank ST. Ear-crease sign of coronary disease. N Engl J Med 1977; 297: 282.

33. Petrakis NL. Diagonal earlobe creases, type A behavior and the death of Emperor Hadrian. West J Med 1980; 132: 87-91.

34. Spaulding CA Jr. Creased earlobe syndrome: a new wrinkle? West J Med 1980; 132: 367.

35. Wyre HW Jr. The diagonal earlobe crease: a cutaneous manifestation of coronary artery disease. Cutis 1979; 23: 328-31.

36. Xu R, Pham J. Frank's sign: a coronary artery disease pre dictor. BMJ Case Rep 2014; 2014: pii: bcr2014205770.

37. Griffing G. Images in clinical medicine. Frank's sign. N Engl J Med 2014; 370: e15.

38. Qamar A, loannides KL, Khetarpal SA, et al. Bilateral earlobe creases and coronary artery disease. Circulation 2014; 130: 92-3.

39. Lichstein E, Chadda KD, Naik D, et al. Diagonal ear-lobe crease: prevalence and implications as a coronary risk factor. N Engl J Med 1974; 290: 615-6.

40. Christiansen JS, Mathiesen B, Andersen AR, et al. Diagonal ear-lobe crease in coronary heart disease. N Engl Jed 1975; 293: 308-9.

41. Lichtstein E, Chapman I, Gupta PK, et al. Diagonal earlobe crease and coronary artery sclerosis. Ann Intern Med 1976; 85: 337-8.

42. Kaukola S. The diagonal ear-lobe crease, a physical sign associated with coronary heart disease. Acta Med Scand Suppl. 1978; 619: 1-49.

43. Kaukola S. The diagonal ear-lobe crease, heredity and coronary heart disease. Acta Med Scand Suppl 1982; 668: 60-3.

44. Doering C, Ruhsenberger C, Phillips DS. Ear lobe creases and heart disease. J Am Geriatr Soc 1977; 25: 183-5.

45. Kristensen BO. Ear-lobe crease and vascular complications in essential hypertension. Lancet 1980; 1: 265.

46. Wermut W, Jaszczenko S, Ruszel A. Ear lobe crease as a risk factor in coronary disease [Polish]. Wiad Lek 1980; 33: $435-8$.
47. Elliott WJ. Ear lobe crease and coronary artery disease. 1,000 patients and review of the literature. Am J Med 1983; 75: 1024-32.

48. Toyosaki N, Tsuchiya M, Hashimoto T, et al. Earlobe crease and coronary heart disease in Japanese. Heart Vessels 1986; 2: 161-5.

49. Gutiu I, el Rifai C, Mallozi M. Relation between diagonal ear lobe crease and ischemic chronic heart disease and the factors of coronary risk. Med Interne 1986; 24 : 111-6.

50. Lesbre JP, Castier B, Tribouilloy C, et al. Frank's sign and coronary disease. Ann Cardiol Angeiol (Paris) 1987; 36: 37-41.

51. Cumberland GD, Riddick L, Vinson R. Earlobe creases and coronary atherosclerosis. The view from forensic pathology. Am J Forensic Med Pathol 1987; 8: 9-11.

52. Patel V, Champ C, Andrews PS, et al. Diagonal earlobe creases and atheromatous disease: a postmortem study. J R Coll Physicians Lond 1992; 26: 274-7.

53. Kirkham N, Murrells T, Melcher DH, et al. Diagonal earlobe creases and fatal cardiovascular disease: a necropsy study. Br Heart J 1989; 61: 361-4.

54. Verma SK, Khamesra R, Bordia A. Ear-lobe crease and ear-canal hair as predictors of coronary artery disease in Indian population. Indian J Chest Dis Allied Sci 1988; 30: 189-96.

55. Nyboe J, Jensen G, Appleyard M, et al. Risk factors for acute myocardial infarction in Copenhagen. I: Hereditary, educational and socioeconomic factors. Copenhagen City Heart Study. Eur Heart J 1989; 10: 910-6.

56. Jensen $G$, Nyboe J, Appleyard $M$, et al. Risk factors for acute myocardial infarction in Copenhagen, II: Smoking, alcohol intake, physical activity, obesity, oral contraception, diabetes, lipids, and blood pressure. Eur Heart J 1991; 12: 298-308.

57. Christoffersen M, Frikke-Schmidt R, Schnohr P, et al. Visible age-related signs and risk of ischemic heart disease in the general population: a prospective cohort study. Circulation 2014; 129: 990-8.

58. Miric D, Rumboldt Z, Pavic M, et al. The role of the diagonal ear lobe crease in the clinical evaluation of coronary risk. Lijec Vjesn 1990; 112: 206-7.

59. Ishii T, Asuwa N, Masuda S, et al. Earlobe crease and atherosclerosis. An autopsy study. J Am Geriatr Soc 1990; 38: 871-6.

60. Elliott WJ, Karrison T. Increased all-cause and cardiac morbidity and mortality associated with the diagonal earlobe crease: a prospective cohort study. Am J Med 1991; 91: 247-54.

61. Elliott WJ, Powell LH. Diagonal earlobe creases and prognosis in patients with suspected coronary artery disease. Am J Med 1996; 100: 205-11.

62. Tranchesi Jr B, Barbosa V, de Albuquerque CP, et al. Diagonal earlobe crease as a marker of the presence and extent of coronary atherosclerosis. Am J Cardiol 1992; 70: 1417-20.

63. Moraes D, McCormack P, Tyrrell J, et al. Ear lobe crease and coronary heart disease. Ir Med J 1992; 85: 131-2.

64. Motamed M, Pelekoudas N. The predictive value of diagonal ear-lobe crease sign. Int J Clin Pract 1998; 52: 305-6.

65. Miric D, Fabijanic D, Giunio L, et al. Dermatological indicators of coronary risk: a case-control study. Int J Cardiol 1998; 67: 251-5.

66. Fabijanic D, Culic V. Diagonal ear lobe crease and coronary artery disease. Am J Cardiol 2012; 110: 1385-6.

67. Kuri M, Hayashi Y, Kagawa K, et al. Evaluation of diagonal earlobe crease as a marker of coronary artery dis- 
ease: the use of this sign in pre-operative assessment. Anaesthesia 2001; 56: 1160-2.

68. Bahcelioglu M, Isik AF, Demirel B, et al. The diagonal ear-lobe crease. As sign of some diseases. Saudi Med J 2005; 26: 947-51.

69. Evrengül H, Dursunoglu D, Kaftan A, et al. Bilateral diag onal earlobe crease and coronary artery disease: a significant association. Dermatology 2004; 209: 271-5.

70. Edston E. The earlobe crease, coronary artery disease, and sudden cardiac death: an autopsy study of 520 individuals. Am J Forensic Med Pathol 2006; 27: 129-33.

71. Lamot SB, Lonegro GG, Hernandez M, et al. Diagonal earlobe crease, a sign of coronary artery disease. Medicina (B Aires) 2007; 67: 321-5.

72. Shmilovich H, Cheng VY, Rajani R, et al. Relation of diag onal ear lobe crease to the presence, extent, and severity of coronary artery disease determined by coronary computed tomography angiography. Am J Cardiol 2012; 109: 1283-7.

73. Shmilovich $H$, Cheng VY, Nakazato R, et al. Incremental value of diagonal earlobe crease to the diamond-forrester classification in estimating the probability of significant coronary artery disease determined by computed tomographic angiography. Am J Cardiol 2014; 114: 1670-5.

74. Kwai-Ping Suen L, Lau YK, Ma HC, et al. Predictive value of auricular diagnosis on coronary heart disease. Evid Based Complement Alternat Med 2012; 2012: 706249.

75. Wu XL, Yang DY, Zhao YS, et al. Diagonal earlobe crease and coronary artery disease in a Chinese population. BMC Cardiovasc Disord 2014; 14: 43.

76. Mehta J, Hamby RI. Diagonal ear-lobe crease as a coronary risk factor. N Engl J Med 1974; 291: 260.

77. Burton JL. Ear-lobe crease. Lancet 1979; 1: 328

78. Rhoads GG, Yano K. Ear-lobe crease and coronary-artery heart disease. Ann Intern Med 1977; 87: 245

79. Rhoads GG, Klein K, Yano K, et al. The earlobe crease: sign of obesity in middle-aged Japanese men. Hawaii Med J 1977; 36: 74-7.

80. Farrell RP, Gilchrist AM. Diagonal ear-lobe crease: an independent risk factor in coronary heart disease? Ulster Med J 1980; 49: 171-2.

81. Jorde LB, Williams RR, Hunt SC. Lack of association of diagonal earlobe crease with other cardiovascular risk factors. West J Med 1984; 140: 220-3.

82. Gibson TC, Ashikaga T. The ear lobe crease sign and coronary artery disease in aortic stenosis. Clin Cardiol 1986; 9: 388-90.

83. Brady PM, Zive MA, Goldberg RJ, et al. A new wrinkle to the earlobe crease. Arch Intern Med 1987; 147: 65-6.

84. Cheng TO. Ear lobe crease and coronary artery disease. J Am Geriatr Soc 1991; 39: 315-6.

85. Cheng TO. Earlobe crease and coronary artery disease: association or coincidence? Am J Med 1992; 93: 587-90.

86. Cheng TO. More research needed on the association between diagonal earlobe crease and coronary artery disease. Arch Intern Med 2000; 160: 2396-7.

87. Kenny DJ, Gilligan D. Ear lobe crease and coronary artery disease in patients undergoing coronary arteriography. Cardiology 1989; 76: 293-8.

88. Eber B, Delgado P. More on the diagonal earlobe crease as a marker of coronary artery disease. Am J Cardiol 1993; 72: 861.

89. Kuon E, Pfahlbusch K, Lang E. The diagonal ear lobe crease for evaluating coronary risk. Z Kardiol 1995; 84: 512-9.
90. Davis TM, Balme M, Jackson D, et al. The diagonal ear lobe crease (Frank's sign) is not associated with coronary artery disease or retinopathy in type 2 diabetes: the Fremantle Diabetes Study. Aust N Z J Med 2000 30: 573-7.

91. Koracevic G, Atanaskovic V. Ear lobe crease: point of disagreement in evidence-based medicine. Am J Forensic Med Pathol 2009; 30: 89.

92. Park JK, Kim HJ, Chang SJ, et al. Risk factors for hemorrhagic stroke in Wonju, Korea. Yonsei Med J 1998 39: 229-35.

93. Zapata-Wainberg G, Vivancos J. Images in clinical medicine: bilateral earlobe creases. N Engl J Med 2013; 368: e32.

94. Muckle TJ. Ear-crease sign and coronary artery disease. Can Med Assoc J 1982; 126: 1373-4.

95. Romoli M, Tordini G, Giommi A. Diagonal ear-lobe crease: possible significance as cardio-vascular risk factor and its relationship to ear-acupuncture. Acupunct Electrother Res 1989; 14: 149-54.

96. Praharaj SK, Sarkar S, Sinha VK. External ear abnormalities in existing scales for minor physical anomalies: are they enough? Psychiatry Res 2012; 198: 324-6.

97. Moncada B, Ruiz JM, Rodriguez E, et al. Ear-lobe crease. Lancet 1979; 1: 220-1.

98. Kobayashi Y, Fukuo Y, Nakazawa Y, et al. The evaluation of the diagonal ear lobe crease (ELC) as a atherosclerotic sign. Nihon Ronen Igakkai Zasshi 1987; 24: 525-31.

99. Chen WC, Zhao TM, Xue W, et al. Ear lobe crease, high serum cholesterol and human leukocyte antigen, risk factors in coronary artery disease. Chin Med J (Engl) 1982; 95: 839-42.

100. Kang EH, Kang HC. Association between earlobe crease and the metabolic syndrome in a cross-sectional study. Epidemiol Health 2012; 34: e2012004.

101. Andresen AR, Christiansen JS, Jensen JK. Diagonal earlobe crease and diabetic retinal angiopathy. N Engl J Med 1976; 294: 1182-3.

102. Raman R, Rani PK, Kulothungan V, et al. Diagonal ear lobe crease in diabetic south Indian population: is it associated with Diabetic Retinopathy? Sankara Nethralaya Diabetic Retinopathy Epidemiology And Molecular-genetics Study (SN-DREAMS, Report no. 3). BMC Ophthalmol 2009; 9: 11.

103. Petrakis NL. Earlobe crease in women: evaluation of reproductive factors, alcohol use, and Quetelet index and relation to atherosclerotic disease. Am J Med 1995; 99: 356-61.

104. Celik S, Erdogan T, Gedikli O, et al. Diagonal ear-lobe crease is associated with carotid intima-media thickness in subjects free of clinical cardiovascular disease. Atherosclerosis 2007; 192: 428-31.

105. Shrestha I, Ohtsuki T, Takahashi T, et al. Diagonal earlobe crease is correlated with atherosclerotic changes in carotid arteries. Circ J 2009; 73: 1945-9.

106. Glavic J, Cerimagic D, Lovrencic-Huzjan A, et al. Frank's sign as a risk factor for cerebrovascular disease. Atherosclerosis 2008; 196: 477-8.

107. Nagatsuka K. Ear-lobe crease as a marker of carotid artery arthrosclerosis. Circ J 2009; 73: 1801-2.

108. Pieri A, Scarpelli P, Rizzo M. Small blood vessel involvement in diabetes mellitus: light microscope study of specimens obtained by ear lobe biopsy. Diabetologia 1965; 1: 109-15

109. Sauty A, Uldry C, Debetaz LF, et al. Differences in PO2 and PCO2 between arterial and arterialized earlobe samples. Eur Respir J 1996; 9: 186-9. 
110. Hughes JM. Blood gas estimations from arterialized capillary blood versus arterial puncture: are they different? Eur Respir J 1996; 9: 184-5.

111. Spiro SG, Dowdeswell IR. Arterialized ear lobe blood samples for blood gas tensions. Br J Dis Chest 1976; 70: 263-8.

112. Korkmaz L, Agac MT, Acar Z, et al. Earlobe crease may provide predictive information on asymptomatic peripheral arterial disease in patients clinically free of atherosclerotic vascular disease. Angiology 2014; 65: 303-7.

113. Ramos PM, Gumieiro JH, Miot HA. Association between ear creases and peripheral arterial disease. Clinics (Sao Paulo) 2010; 65: 1325-7.

114. Choi SI, Kang HC, Kim CO, et al. Relationship between earlobe crease and brachial-ankle pulse wave velocity in non-hypertensive, non-diabetic adults in Korea. Epidemiol Health 2009; 31: e2009002.

115. Korkmaz L, Agac MT, Erkan H, et al. Association between diagonal earlobe crease and cardio-ankle vascular index in asymptomatic hypertensive patients. Med Princ Pract 2013; 22: 530-4.

116. Singh M, Bedi US. Is atherosclerosis regression a realistic goal of statin therapy and what does that mean? Curr Atheroscler Rep 2013; 15: 294.

117. Rizzo M, Nikolic D, Banach M, et al. Statin treatment in the elderly: how much do we know? Arch Med Sci 2013; 9: 585-8.

118. Lucenteforte E, Romoli M, Zagli G, et al. Ear lobe crease as a marker of coronary artery disease: a meta-analysis. Int J Cardiol 2014; 175: 171-5.

119. Athyros VG, Kakafika Al, Karagiannis A, et al. Do we need to consider inflammatory markers when we treat atherosclerotic disease? Atherosclerosis 2008; 200: 1-12.

120. Sinha N, Devabhaktuni S, Kadambi A, et al. Can echocardiographically estimated pulmonary arterial elastance be a non-invasive predictor of pulmonary vascular resistance? Arch Med Sci 2014; 10: 692-700.

121. Gluba-Brzozka A, Michalska-Kasiczak M, Franczyk-Skora B, et al. Markers of increased cardiovascular risk in patients with chronic kidney disease. Lipids Health Dis 2014; 13: 135 